\title{
A New Chaotic Mixer Design Based on the Delta Robot and Its Experimental Studies
}

\author{
Onur Kalayci $\mathbb{D}^{1},{ }^{1}$ Ihsan Pehlivan, ${ }^{2}$ Akif Akgul, ${ }^{3}$ Selcuk Coskun, ${ }^{4}$ and Ersin Kurt ${ }^{5}$ \\ ${ }^{1}$ Faculty of Technology, Department of Mechatronics Engineering, Sakarya University of Applied Sciences, Adapazari, \\ Sakarya 54050, Turkey \\ ${ }^{2}$ Faculty of Technology, Department of Electric-Electronics Engineering, Sakarya University of Applied Sciences, Adapazari, \\ Sakarya 54050, Turkey \\ ${ }^{3}$ Faculty of Engineering, Department of Computer Engineering, Hitit University, Corum 19030, Turkey \\ ${ }^{4}$ IMKB Sakarya Vocational and Technical Anatolian High School, Adapazari, Sakarya 54050, Turkey \\ ${ }^{5}$ Faculty of Electric-Electronics Engineering Afyon Kocatepe University, Afyon 03200, Turkey
}

Correspondence should be addressed to Onur Kalayci; okalayci67@gmail.com

Received 20 December 2020; Revised 23 January 2021; Accepted 7 March 2021; Published 23 March 2021

Academic Editor: Zenghui Wang

Copyright $(2021$ Onur Kalayci et al. This is an open access article distributed under the Creative Commons Attribution License, which permits unrestricted use, distribution, and reproduction in any medium, provided the original work is properly cited.

\begin{abstract}
In this study, a new chaotic mixer based on the Delta robot was designed and produced which had been controlled with Arduino Uno card and MATLAB. First of all, chaotic mixing systems with different dynamic properties were chosen for the chaotic mixing process. Then, by solving the chaotic systems selected in the MATLAB with the Runge Kutta 45 (RK45) numerical solution algorithm, the results in the integer format were obtained. The obtained chaotic time-series results were transformed into 3dimensional position information for the servomotors used in the mixer with the algorithm developed in MATLAB. The supervision was provided to ensure that the newly designed chaotic mixer was pacing chaotically in $x, y$, and $z$ coordinates by transferring the chaotic position information to the Arduino Uno R3 card via USB 2.0. With the software developed in MATLAB, the performances of 7 diversified chaotic systems' trajectories and circular motion trajectories were compared over the numerical simulation orbital distribution ratio (ODR). In the final stage, in a solid-liquid mixture type, at the selected constant mixing time, experimental studies were performed where homogeneity and orbital distribution ratio (ODR) parameters were compared by using 7 diversified chaotic systems. The designed and produced chaotic mixer can also be used in experimental studies of certain liquid-liquid mixture types. It is thought that this prototype presented in the article will serve the aim of developing new chaotic mixer systems and algorithms to derive more homogeneous mixtures in a shorter time.
\end{abstract}

\section{Introduction}

Combining at least two different substances is called mixing, and the devices that carry out this process are called mixers. The substances required to be mixed are in the form of solid, liquid, gas, or varieties of those. The most emphasized parameters in mixing are the duration and homogeneity of the mixing process. The higher the homogeneity of the mixture and the shorter the working time of the mixer, the higher the performance of the mixer is [1].

Mixers are used in several areas such as at homes, in kitchens, in bakeries with dough kneading machines, in factories, and in construction zones. The development of more widely used mixers and designing more efficient mixers are of great importance for the companies that manufacture these machines or the ones that use these machines [1].

Factors affecting mixing are given in the following: mixing temperature, mixing duration, agitator type, and agitator motor speed. Mixer types used in industries are given in the following: turbine-type stirrer, propeller mixer, flat-blade turbine type, kneader, dry mixer, and planetary mixer [1].

Professors Banhero and Bodger [2] researched mixing theory of matter states in solid, liquid, and gaseous in their study. Vauck and Müller [3] studied on the degree of the 
mixture, mixing duration, mixing methods, and standardization of mixers. Henzler and Eignung [4] observed the homogeneity of the continuous mixers in their study. Ilten examined mixers, mixing methods, mixing events, and mixer power calculations in his thesis study [5]. Can, in his thesis study, modelled the homogeneous mixing of two different fluids with a disc axially moving back and forth in a cylindrical container and rotating at a certain speed around its axis [6].

Inspiring studies such as machining sequences optimization [7], automotive testing [8], navigation control and stability investigation of a mobile robot [9], and complex trajectories of the omniwheel robot in machine [10] in automotive and robotic fields have been carried on in recent years.

In 1988, Angeles designed the Delta robot at the Lausanne Federal Polytechnic Institute (EPFL). He designed this robot to perform translational movements in the $3 \mathrm{D}$ space [11]. In 2004, Zsombor-Murray wrote a software that could analytically model the forward and inverse kinematics of the delta robot, the intersection points of a central fixed circle, and a sphere with a central motion and make these calculations on a computer [12]. Şanlitürk designed the Delta robot which can do the visual operation, and he searched its performance features in his thesis study [13]. Qiaoling et al., in their study, suggested the optimal design method of a Linear Delta robot (LDR) to obtain the proposed rectangular working area (PCDW) [14]. Xin-Jun et al. presented a new design method by considering the desired working area and the swing range in the spherical joints of a Delta robot in their study [15].

There are many scientific studies on chaotic systems, chaotic signals, and engineering applications in the literature [16-27]. One of the application areas where chaotic signals are used has been chaos-based mixers. Chau et al. used a floating DC engine as a mixer in their study. The speed of the DC motor is chaotically adjusted, and the mixing is done with time delay [28]. Ye and Chau used a DC engine as a mixer in their study. The speed of the DC engine was chaotically adjusted by the unstable method [29]. Ye and Chau used a time-delayed floating DC engine as a mixer in their study [30]. In all three studies, the results of chaotic mixing were compared with the results of mixing at constant speed. The acid-base neutralization reaction was evaluated in these experiments [28-30]. Murtadha et al. studied on the chaotic control of a liquid mixer. In this study, the water-salt mixture was stirred for 30 seconds. The results were evaluated by making concentrated measurements [31]. In Zhang and Chen's study, the vessel containing the mixture was fixed to a plate rotated by a DC motor by keeping the blades of the mixer fixed. The motor speed was chaotically adjusted by using the Chua circuit. In the experiment, the water-sugar mixture was evaluated [32]. Kavur et al. designed a chaotic system-based Delta robot in their study to mix graphene nanoplatelets [33].

In the second part of the study, the design of the delta robot-based chaotic mixer and the conversion calculations of the instantaneous signal amplitude values of the chaotic time series $(x, y, z)$ produced by the new software developed in MATLAB to the angle values of the Delta robot arms were explained. In the third part of the study, the performances of the orbits and circular motion trajectories of 7 different chaotic systems are evaluated by comparing them with the numerical simulation orbital distribution ratios obtained in MATLAB. In the fourth part of the study, experimental studies have been carried out for the Sprott_A chaotic system with the highest orbital distribution ratio and the Lu-Chen chaotic system with the lowest orbital distribution ratio and the movement of the mixer propeller in a circular and fixed position.

\section{Materials and Methods}

2.1. Chaotic Systems. Chaos is a branch of science that helps explain nonlinear events, expressed as the order of disorder. Chaos is not a random event, as it has its own internal order in addition to complex behaviors. Chaos can be expressed as the most complex known state of dynamical systems. Chaos focuses on the interrelationship of events that occur from delicate differences within randomly thought situations. Chaos science is a branch of science that tries to understand the events in real life that are thought to behave randomly such as the movement of clouds, the cigarette smoke movement, bubbling river movements, and the movement of water flowing from the tap $[34,35]$.

Chaos can be briefly expressed as dynamical systems that are hypersensitive to initial and input conditions [34, 36]. Due to this sensitivity of chaotic systems to the initial and input conditions, small changes in these values cause the output of the system to change. For this reason, although chaotic structures are deterministic systems, the behavior of the system can be predicted only for a short time. During the following iterations, the behavior of chaotic systems becomes unpredictable $[34,35]$. Due to these characteristics, the number of chaotic system studies conducted in scientific and industrial areas such as cryptology, control, image processing, communication, and artificial neural networks is increasing rapidly $[34,37,38]$.

Chaotic systems can be studied in two groups as discretetime and continuous-time chaotic systems. Discrete-time chaotic systems are usually unidimensional or bidimensional which means they can consist of one or two equations. On the contrary, continuous-time chaotic systems are at least tridimensional which means they contain at least three equations $[34,39,40]$.

2.1.1. Discrete-Time Chaotic Systems. Discrete-time chaotic systems are formed by the iteration of a proper nonlinear function; in other words, they are the chaotic systems with the feedback property. Discrete-time chaotic systems can be used directly in desired applications in digital environments without the need for discretization algorithms such as continuous-time chaotic systems [41].

There are many unidimensional and bidimensional discrete-time chaotic systems in the literature [34, 38, 40]. Logistic Map [42], Cubic Map [43], Sine Map [44], and Tent Map [45] chaotic systems can be shown as examples of 
unidimensional discrete-time chaotic systems. Henon Map [46], Lozi Map [47], Burgers' Map [48], Discrete PredatorPrey Map [49], and Arnold's Cat Map [50] can be shown as examples of bidimensional discrete-time chaotic systems [41].

\subsubsection{Continuous-Time Chaotic Systems.} Continuous-time chaotic systems (CTCS) are generally expressed by ordinary differential equations. $N$ continuoustime first-order ordinary differential equations with $I=1,2$, $3, \ldots, n$ can be like the following equation $[34,51]$ :

$$
\begin{aligned}
& \frac{\mathrm{d} x^{(i)}}{\mathrm{d} t}=f_{1}\left(x^{(i)}, x^{(i+1)}, \ldots, x^{(n)}\right) \\
& \frac{\mathrm{d} x^{(i+1)}}{\mathrm{d} t}=f_{2}\left(x^{(i)}, x^{(i+1)}, \ldots, x^{(n)}\right) . \\
& \vdots \\
& \frac{\mathrm{d} x^{(n)}}{\mathrm{d} t}=f_{n}\left(x^{(i)}, x^{(i+1)}, \ldots, x^{(n)}\right)
\end{aligned}
$$

Ordinary differential equations can be in the vector form as in equation (2) if the above expressions are edited:

$$
\begin{aligned}
& \frac{\mathrm{d} x(t)}{\mathrm{d} t}=F[x(t)], \\
& x\left(t_{0}\right)=x_{0} .
\end{aligned}
$$

Given in the equation, $x$ is an $n$-dimensional vector, while $x_{0}$ means the initial state vector and $t$ means the time. Discrete-time chaotic systems are expressed by unidimensional nonlinear simple equations, whereas continuous-time chaotic systems are at least 3-dimensional. So, the expression $n$ must be at least three $[34,51]$.

In the literature, Zhang et al. [52], Precup et al. [53], Sheu et al. [54], Kuetche et al. [55], Sundarapandian [56], Chen and Ueta [57], Vembarasan and Balasubramaniam [58], and Rucklidge $[59,60]$ chaotic systems can be shown as an example of continuous-time chaotic systems; Arneodo [61], Hindmarsh-Rose [62], and Spring [63] chaotic systems can be shown as an example of hyperchaotic systems [35].

2.2. Delta Robot. Recently, the demand for high-speed pickup and packaging systems has been increasing in the production areas of industry. Nowadays, parallel manipulators have become more significant as parallel manipulators are more robust and have more precise positioning due to highprecision motion performance is required from many mechanical systems. The delta robot, one of the parallel manipulators in Figure 1, can move in the required $x, y$, and $z$ coordinates of the working area with 3 motors mounted on a fixed plate and the plates attached to each. The motors on the fixed plate are placed symmetrically, and each motor with the connected arm are controlled. For this reason, stationary and mobile planes constantly remain parallel. Therefore, the Delta robot moves as 3 axes $[64,65]$.

2.3. The Design of the Chaotic Delta Robot. Servomotors with $5 \mathrm{VDC}$ and $10 \mathrm{Nm}$ torque were preferred for the movement of Delta robot parallel arms. The Arduino Uno R3 control card was chosen for the control of the designed delta robot. A $12 \mathrm{~V} \mathrm{DC} 12 \mathrm{rpm}$ motor was used for the mixer motor connected to the Delta robot. PWM (Pulse Width Modulation) based DC motor speed control was designed and used for speed control of the mixer motor. Mechanical parts were made of the lightweight and durable aluminium material. All components related to the design of the Delta robot-based chaotic mixer can generally be seen in Figure 2 .

A standard Arduino software package, which enables the communication between MATLAB and Arduino, can be downloaded and installed from MATLAB's website. With the help of the Arduino software package, the chaotic location information produced by the algorithm developed in MATLAB is sent to the Arduino Uno R3 card on the Delta robot. The DC motor can be operated at the desired speed and direction with the potentiometer on the PWM DC motor control card at the same time. The circuit connection diagram of the Delta robot-based chaotic mixer is shown in Figure 3.

The designed and implemented Delta robot-based chaotic mixer is shown in Figure 4.

2.4. Chaotic Mixer Software. The block diagram of Delta robot-based chaotic mixer software and components is shown in Figure 5.

As shown in the block diagram in Figure 5, the Arduino software package downloaded from the MATLAB web enabled the communication between the Arduino Uno R3 card which was connected to servo motors and MATLAB programme. In the MATLAB platform, an interface was developed in which chaotic system, numerical solution iteration number, and servo motor speeds could be selected and changed. One of 7 different chaotic systems can be chosen through the interface. $x, y$, and $z$ chaotic time series data are obtained by solving the chaotic system selected from the developed interface with the method of the RK45 numerical solution in MATLAB.

2.4.1. Conversion of Instant Signal Amplitude Values to Robot Angle Values. With the new software developed in the MATLAB platform, maximum signal amplitude value (Max_SAV) and minimum signal amplitude value (Min_SAV) are determined by generating instant signal amplitude values (I_SAV) of the chaotic time series $(x, y, z)$.

Max_SAV is linearly scaled to correspond to the maximum mobility angle value of the robot arms (Max_MAV), while Min_SAV is linearly scaled to correspond to the minimum mobility angle value of the robot arms (Min_$\mathrm{MAV}$ ) and converted to chaotic position information (robot angle values (R_AV)). 


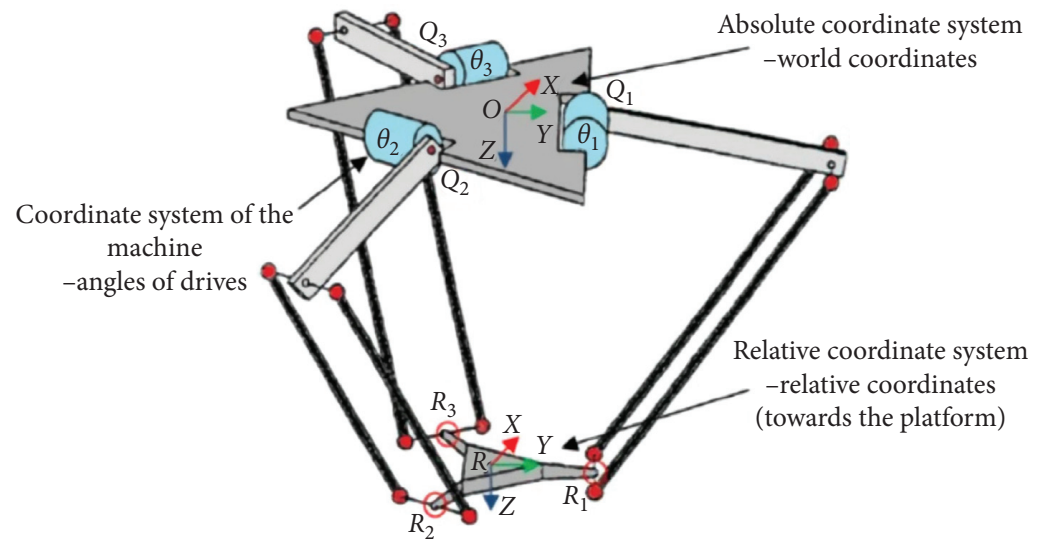

Figure 1: Delta robot [64].

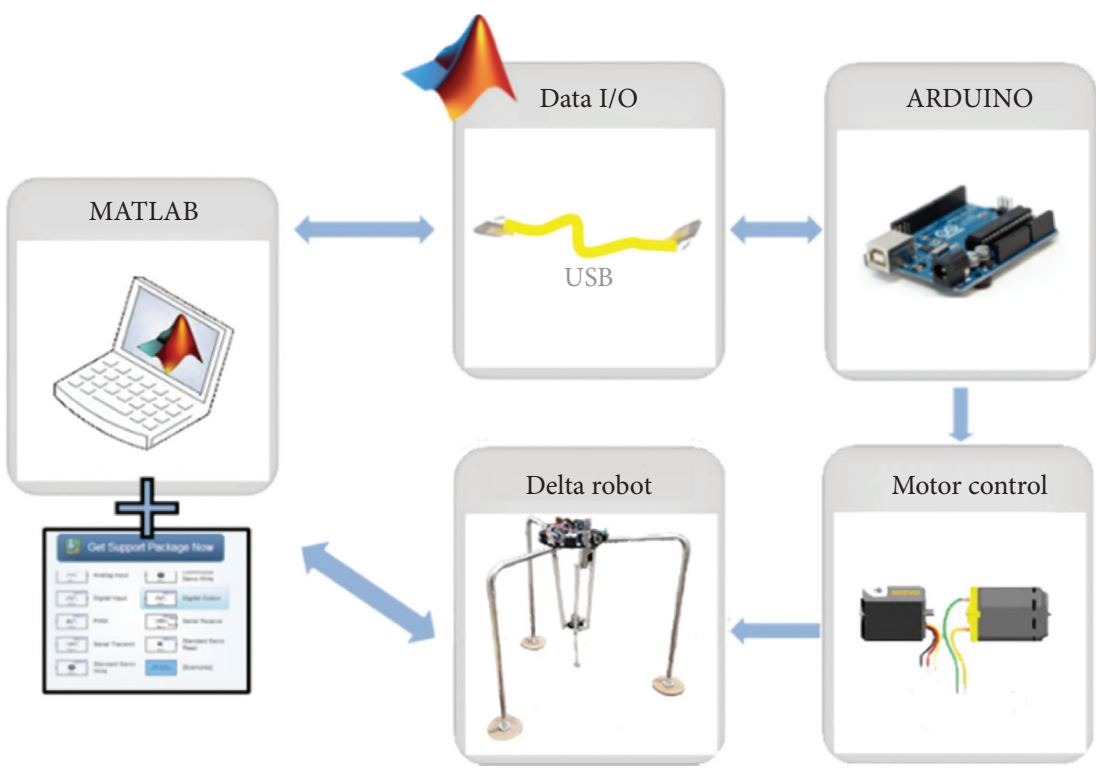

Figure 2: The components related to the design of Delta robot stages.

The variants and abbreviations used in the algorithm that converts the I_SAV $(x, y, z)$ of chaotic time series to R_AV by linear scaling are shown in Table 1 .

Max_MAV and Min_MAV can be entered with the interface in the program. The robot arms of the newly designed delta robot-based chaotic mixer can move up to $144^{\circ}$ on each axis $(x, y, z)$ due to their mechanical properties. Therefore, Max_MAV and Min_MAV were, respectively, entered as $0^{\circ}$ and $144^{\circ}$ via the interface.

The technical details of the algorithm that converts I_SAV to linear scale R_AV during the operation of the mixer system are as follows:

(a) Min_SAV and Max_SAV are determined for each of the chaotic time series signals $(x, y, z)$

(b) By substracting Min_SAV from Max_SAV, peak-topeak signal amplitude value (PP_SAV) is found as in the following equation:

$$
\text { PP_SAV }=\text { Max_SAV }- \text { Min_SAV. }
$$

(c) By dividing Max_MAV (e.g., 144 ) entered through the interface into PP_SAV, the angle value per unit amplitude (PUA_AV) is calculated as in the following equation:

$$
\text { PUA_AV }=\frac{\text { Max_MAV }}{\text { PP_SAV }} .
$$

(d) The result found by subtracting Min_SAVs from the chaotic I_SAV is multiplied by PUA_AV to obtain the required robot angle values (R_AV) as in the following equation:

$$
\text { R_AV }=(\text { I_SAV }- \text { Min_SAV }) x \text { PUA_AV. }
$$

Examples of algorithms that convert I_SAV on $X$-axis, $Y$-axis, and $Z$-axis to linear scale $\mathrm{R} \_\mathrm{AV}$ during the operation of the mixer system are shown in Figures 6-8.

The mixer propeller connected to the parallel arms of the Delta robot moves in the 3-dimensional chaotic positions of the selected system, as shown in Figure 9. 


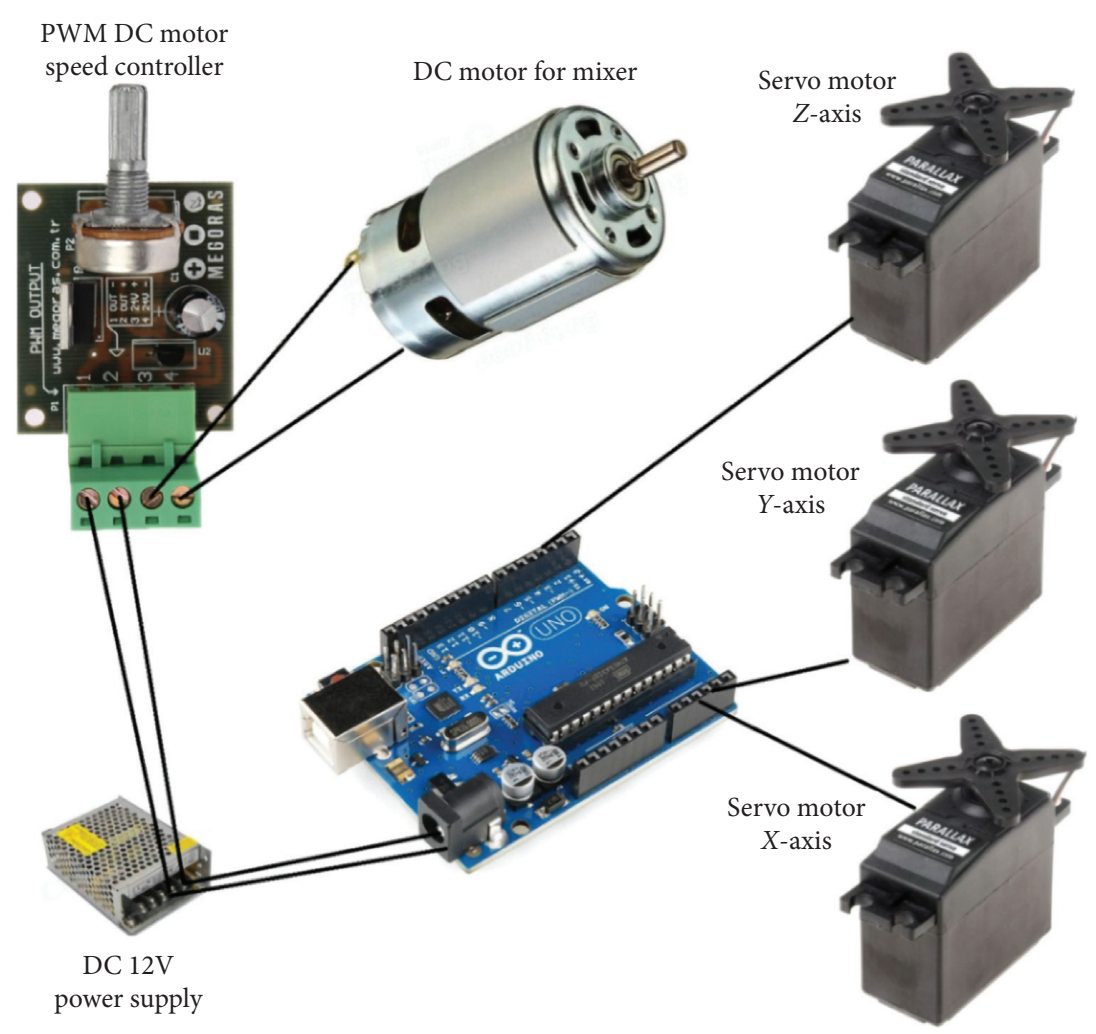

Figure 3: The circuit connection diagram of the delta robot-based chaotic mixer.

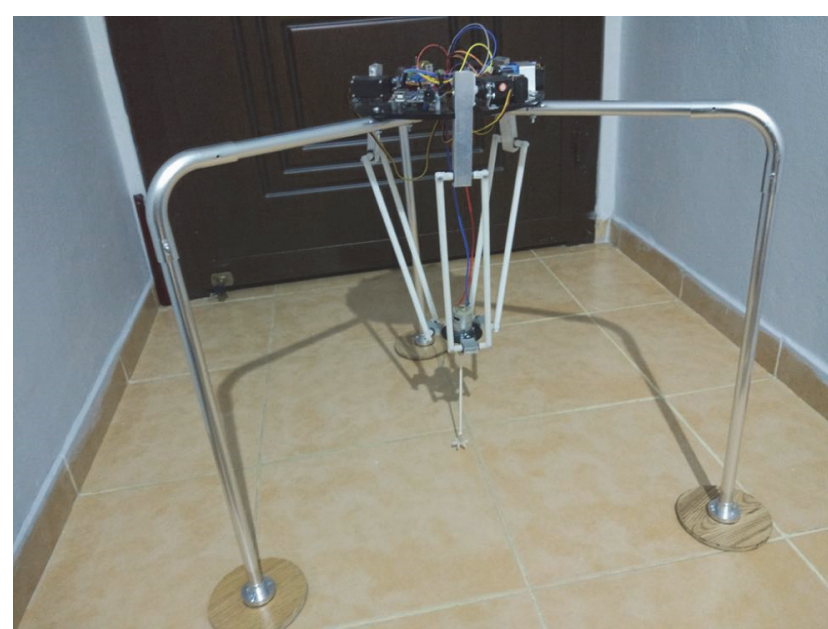

FIGURE 4: Designed delta robot-based chaotic mixer.

2.4.2. Mixer Propeller's Motion Zones in the Phase Space. The orbital distribution rate (ODR) is calculated by dividing the three-dimensional phase space into 8 equal regions where the chaotic propeller mixer will be travelling. The area where the chaotic mixer propeller travels is divided into 8 equal zones in the cubic form, as shown in Figure 10.

Within the software, the counters that will count the number of points in which the chosen chaotic system trajectory passes through 8 zones are reset. When the selected chaotic system trajectory passes through any of the regions in Figure 8, the counter for that zone within the software is incremented by one. This operation is repeated as the amount of numerical solution iterations' numbers entered through the interface. Since the Max_MAV and Min_MAV are entered as $0^{\circ}$ and $144^{\circ}$, respectively, the position angle values of the chaotic propeller mixer in 8 different regions have become as in Table 2 .

When the numerical solution iteration number chosen entered through the interface for the chosen chaotic system is completed, the number and percentage of the trajectory existence in each region are printed on the screen with the software. Sample tables belonging to the percentages of the chaotic system trajectories' presence in each region have been shown in Section 3.2. Also, the percentages of the chaotic system's trajectories presence in each region were used to calculate the orbital distribution rate (ODR) in Section 2.4.3.

2.4.3. Calculation of the Orbital Distribution Rate. To compare the orbital distribution performance of chaotic systems, it was intended to calculate the uniformity degree of the orbital distribution ratio (ODR) with great accuracy by using the trajectory percentages in each of the 8 different zones.

Equation 6 was used for the calculation of the orbital distribution rate (ODR): 

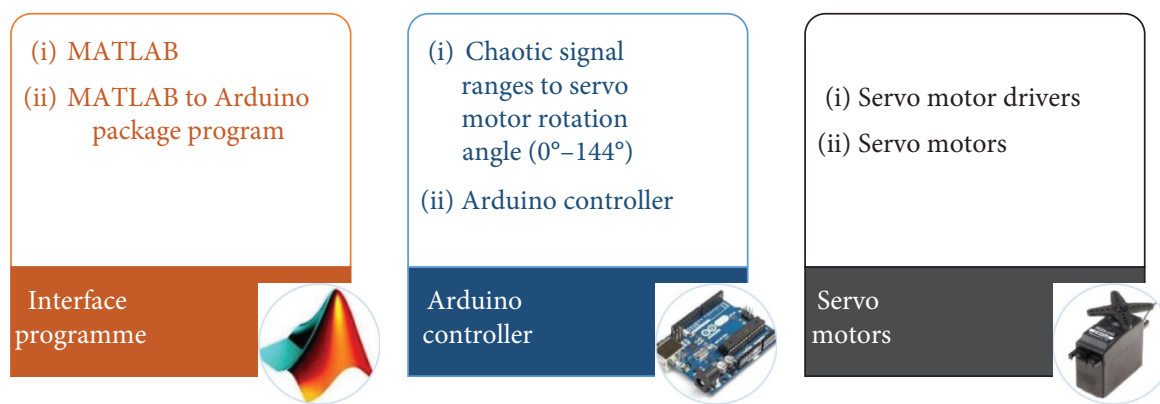

Figure 5: Block diagram of the Delta robot-based chaotic mixer.

TABLE 1: Variants and abbreviations used in the algorithm that converts I_SAV of chaotic time series to $(x, y, z)$ to R_AV by scaling linearly.

\begin{tabular}{lc}
\hline Variants & Abbreviations \\
\hline Maximum mobility angle value of robot arms & Max_MAV \\
Minimum mobility angle value of robot arms & Min_MAV \\
Instant signal amplitude value & I_SAV \\
Minimum signal amplitude value & Min_SAV \\
Maximum signal amplitude value & Max_SAV \\
Peak-to-peak signal amplitude value & PP_SAV \\
Angle value per unit amplitude & PUA_AV \\
Robot angle values & R_AV \\
\hline
\end{tabular}

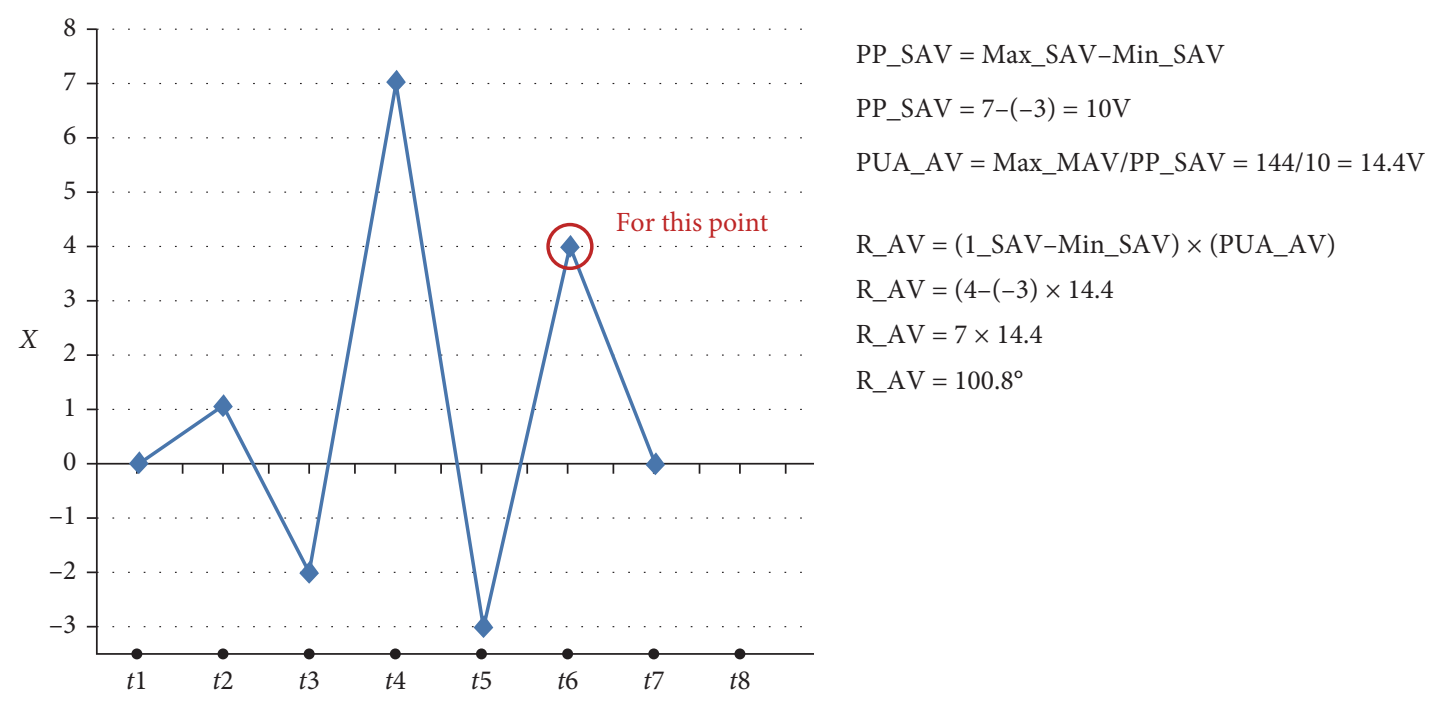

FIgURE 6: The example of the algorithm that converts I_SAV in the $X$-axis to linear scale R_AV during the operation of the mixer system.

$\mathrm{ODR}=100-\left(\frac{(\mid 12,5-\text { Zone1 }|+| 12,5-\text { Zone2 }|+| 12,5-\text { Zone3 }|+| 12,5-\text { Zone4 }|+| 12,5-\text { Zone } 5|+| 12,5-\text { Zone6 }|+| 12,5-\text { Zone7 }|+| 12,5-\text { Zone } 8 \mid)}{1.75}\right)$

In the design of this formula, if the trajectories of a chaotic system in 8 zones are equal in number, the ODR is demanded to be $100 \%$. For example, in a system with $100 \%$, the rate of presence in each of the 8 zones is $12.5 \%$. If the rate of being found in each zone (12.5\%) is put in equation (4), it can be seen that ODR is calculated as $100 \%$.

On the contrary, for a system with $0 \%$ ODR, it is thought that the trajectories of the chaotic system are collected in only 1 zone and never found in the other 7 zones. For example, in a system with an ODR of $0 \%$, the rate of presence in only one zone is $100 \%$, whereas the rate of occurrence in the other 7 zones is $0 \%$. When these ratios are replaced in equation (4), it can be seen that the ODR is calculated as $0 \%$.

The ODRs of the chaotic systems examined in the chaotic mixer are shown by charts in Section 3.2. 


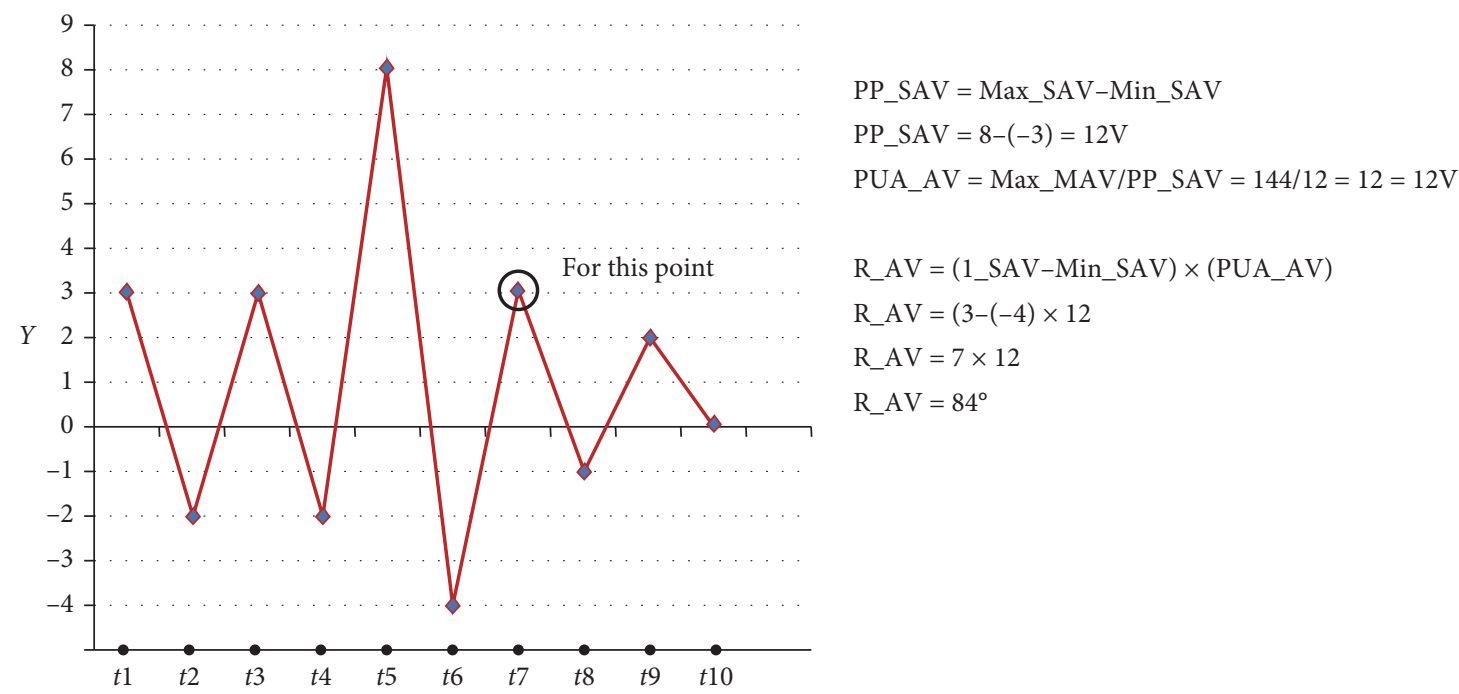

FIGURE 7: The example of the algorithm that converts I_SAV in the $Y$-axis to linear scale R_AV during the operation of the mixer system.

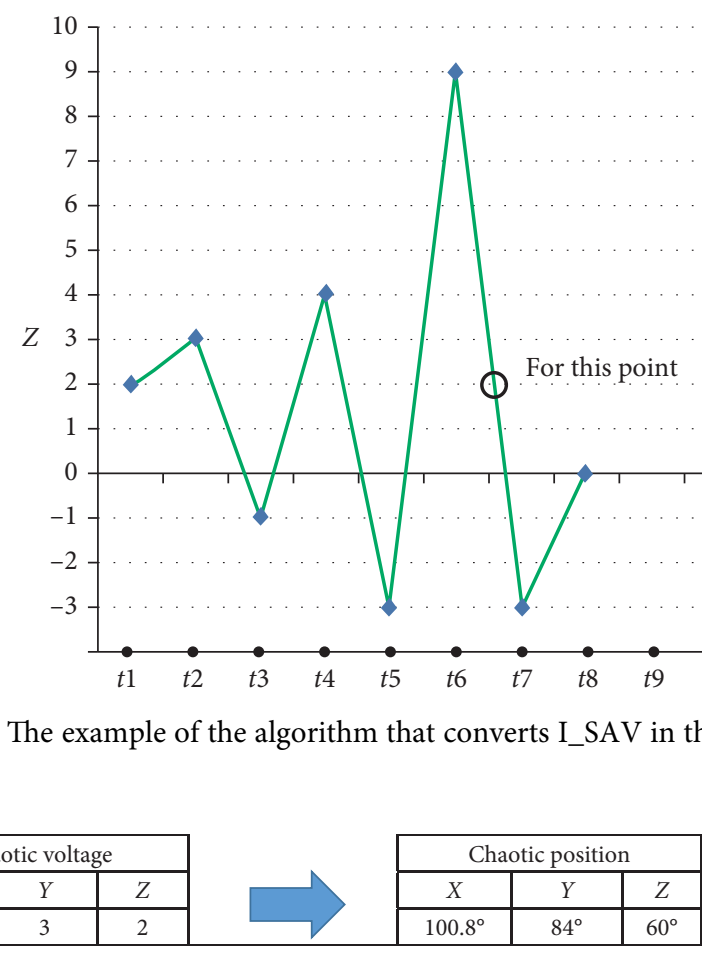

Figure 9: The $X, Y$, and $Z$ axis of the mixer system during operation I_SAV converted to linear scale R_AV.

\section{Comparison of Selected Chaotic Systems and Orbital Distribution Ratios}

7 different chaotic systems with different dynamic features were preferred to investigate their potential to be used in the new Delta robot-based chaotic mixer. These systems can be chosen through the interface program. The performances of the trajectories and circular motion trajectories of 7 different chaotic systems were evaluated by comparing them to the numerical simulation ODRs obtained in the MATLAB platform. The differential equations of the selected sample chaotic systems are given below.

\subsection{Selected Chaotic Systems}

3.1.1. Sprott A System. The initial conditions were taken as $x$ $(0)=0, y(0)=0.5$, and $z(0)=0$ in the differential equation of the Sprott94_A chaotic attractor [A] seen in the following equation:

$$
\begin{aligned}
& x=y \\
& y=-x+y \cdot z, \\
& z=1-y^{2} .
\end{aligned}
$$




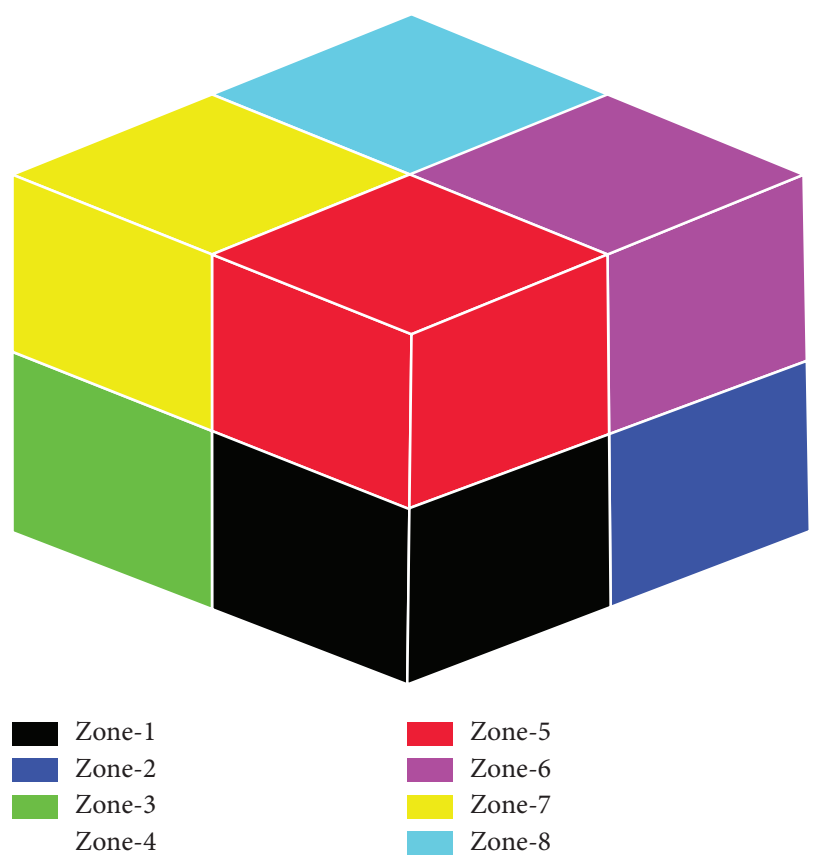

Figure 10: The zones where the chaotic propeller mixer is moving.

TABLe 2: The position angle values of the chaotic propeller mixer on 8 different regions (in degrees).

\begin{tabular}{|c|c|c|c|}
\hline Zones & Pos_X & Pos_Y & Pos_Z \\
\hline Zone-1 & $0 \leq$ pos_ $X<72$ & $0<$ pos_Y $Y \leq 72$ & $0<$ pos_ $Z \leq 72$ \\
\hline Zone-2 & $72 \leq$ pos_ $X<144$ & $0<$ pos_Y $Y \leq 72$ & $0<$ pos_ $Z \leq 72$ \\
\hline Zone-3 & $0 \leq$ pos_ $\_<72$ & $72<$ pos_Y $Y \leq 144$ & $0<$ pos $\_Z \leq 72$ \\
\hline Zone-4 & $72 \leq$ pos_ $\_<144$ & $72<$ pos_Y $\leq 144$ & $0<$ pos $\_Z \leq 72$ \\
\hline Zone-5 & $0 \leq$ pos_ $X<72$ & $0<$ pos_Y $Y \leq 72$ & $72<$ pos_ $Z \leq 144$ \\
\hline Zone-6 & $72 \leq$ pos_ $\_X<144$ & $0<$ pos_Y $Y \leq 72$ & $72<$ pos_ $Z \leq 144$ \\
\hline Zone-7 & $0 \leq$ pos_ $X<72$ & $72<$ pos_Y $\leq 144$ & $72<$ pos_ $Z \leq 144$ \\
\hline Zone- 8 & $72 \leq$ pos_ $X<144$ & $72<$ pos_Y $Y \leq 144$ & $72<$ pos_ $Z \leq 144$ \\
\hline
\end{tabular}

3.1.2. Pehlivan-Wei System. The initial conditions were taken as $x(0)=-4, y(0)=1$, and $z(0)=-4$ in the differential equation of the Pehlivan-Wei chaotic attractor [B] seen in the following equation:

$$
\begin{aligned}
& x=y-y \cdot z, \\
& y=y+y \cdot z-2 \cdot x, \\
& z=2-x \cdot y-y^{2} .
\end{aligned}
$$

3.1.3. Aizawa System. The initial conditions were taken as $x$ $(0)=0.1, y(0)=0 \mathrm{ve}$, and $z(0)=0$ in the differential equation of the Aizawa chaotic attractor $[\mathrm{C}]$ seen in the following equation:

$$
\begin{aligned}
& x=(z-0.7) \cdot x-3.5 \cdot y \\
& y=3.5 \cdot x+(z-0.7) \cdot y \\
& z=0.6+0.95 \cdot z-\frac{z^{3}}{3}-\left(x^{2}+y^{2}\right) \cdot(1+0.25 \cdot z)+0.1 \cdot z \cdot x^{3} .
\end{aligned}
$$

3.1.4. Guckenheimer-Holmes System. The initial conditions were taken as $x(0)=1, y(0)=-1$ ve, and $z(0)=1$ in the differential equation of the Guckenheimer-Holmes chaotic attractor [D] seen in the following equation:

$$
\begin{aligned}
& x=0.4 \cdot x-20.25 \cdot y+3 \cdot z \cdot x+1.6 \cdot z \cdot\left(x^{2}+y^{2}\right) \\
& y=0.4 \cdot y+20.25 \cdot x+3 \cdot z \cdot y \\
& z=1.7-z^{2}-0.44 \cdot\left(x^{2}+y^{2}\right)-0.4 \cdot z^{3} .
\end{aligned}
$$

3.1.5. Lu-Chen System. The initial conditions were taken as $x(0)=-3, y(0)=0$ ve, and $z(0)=3$ in the differential equation of the $\mathrm{Lu}-\mathrm{Chen}$ chaotic attractor $[\mathrm{A}]$ seen in the following equation:

$$
\begin{aligned}
& x=5 \cdot x-y \cdot z, \\
& y=-10 \cdot y+x \cdot z, \\
& z=-3.4 \cdot z+x \cdot y .
\end{aligned}
$$


3.1.6. Hadley System. The initial conditions were taken as $x$ $(0)=0, y(0)=0 \mathrm{ve}$, and $z(0)=1.3$ in the differential equation of the Hadley chaotic attractor [A] seen in the following equation:

$$
\begin{aligned}
& x=-y^{2}-z^{2}-0.25 \cdot x+2, \\
& y=x \cdot y-4 \cdot x \cdot z-y+1, \\
& z=4 \cdot x \cdot y+x \cdot z-z
\end{aligned}
$$

3.1.7. Halvorsen System. The initial conditions were taken as $x(0)=-5, y(0)=0$ ve, and $z(0)=0$ in the differential equation of the Halvorsen chaotic attractor $[\mathrm{A}]$ seen in the following equation:

$$
\begin{aligned}
& x=-1.27 \cdot x-4 \cdot y-4 \cdot z-y^{2} \\
& y=-1.27 \cdot y-4 \cdot z-4 \cdot x-z^{2} \\
& z=-1.27 \cdot z-4 \cdot x-4 \cdot y-x^{2}
\end{aligned}
$$

\subsection{Comparison of Chaotic Systems and Circular-Motion} Orbital Distribution Rates. For 7 different chaotic systems and circular motion developed in the MATLAB platform, the orbital distribution rates (ODR) obtained at the end of the same numerical solution iterations entered through the software were calculated as in Table 3. According to Table 4, the Sprott_A system has been the chaotic system which has the highest ODR with $93.7 \%$. The Lu-Chen system is the chaotic system which has the lowest ODR with $40.1 \%$. The ODR values belonging to other chaotic systems were found between ODR values belonging to the Sprott_A and LuChen systems, as shown in Table 3. The ODR obtained for the circular motion is shown as $42.9 \%$.

The calculation data of the Sprott_A system having the highest ODR were given in Section 3.2.1, the calculation data of the Lu-Chen system having the lowest ODR were given in Section 3.2.2, and the data of the ODR calculation for the circular motion was given in Section 3.2.3. Since the ODR data of the other systems were not used in experimental studies, they have not been presented in this article.

3.2.1. ODR Data of the Sprott_A System. The time series for the Sprott_A chaotic system obtained with the software developed in the MATLAB platform are shown in Figure 11, and the three-dimensional phase portraits are shown in Figures 12-14.

ODR calculation software was developed in the MATLAB platform, and the orbital distribution rate belonging to the Sprott_A chaotic system was calculated as seen in Table 4.

3.2.2. ODR Data of the Lu-Chen System. The time series obtained for the Lu-Chen chaotic system with the software developed in the MATLAB platform are shown in Figure 15, and 3-dimensional phase portraits are shown in Figures 16-18.
The orbital distribution ratio (ODR) of the $\mathrm{Lu}-\mathrm{Chen}$ chaotic system was calculated as in Table 5 with ODR calculation software developed in the MATLAB platform.

3.2.3. Circular Motion ODR. The time series obtained for the circular motion with the software developed in the MATLAB platform is shown in Figure 19, while the three-dimensional phase portraits are shown in Figures 20-22.

The orbital distribution ratio (ODR) for the orbital motion was calculated with the ODR calculation software developed in the MATLAB platform as seen in Table 6.

\section{Mixing Experiments}

A mixture of water and sugar was preferred as the solution to be used in mixing experiments. Due to the physical structure of the chaotic mixer, 19.5 liters of water and 0.5 liters of sugar, in total 20 liters of solutions were prepared in the cubic mixing vessel for each experiment. The mixing experiments were performed first for the Sprott_A chaotic system with the highest ODR and the Lu-Chen chaotic system with the lowest ODR. Then, the experiments were repeated for the circular and constant position motion of the propeller mixer. For 4 different experimental studies, a 5-minute period was selected from the interface programme.

4.1. Homogeneity Tests of Mixtures. The MA871 digital Brix refractometer, seen in Figure 23, was used for the homogeneity test of the sugar-water mixture. MA871 is an optical device using refractive index measurement to determine the amount of soluble dry matter (Brix) as a percentage in aqueous solutions. The method is both simple and fast. Samples are measured after a simple user calibration with deionized or distilled water. The meter can measure the refractive index of the sample in a few seconds and convert it to a Brix concentration unit [66].

Since 19,500 grams of water and 500 grams of sugar were used for the sugar-water mixture, the maximum sugar ratio that can be measured with the measuring device for this mixture has been $500 /(19500+500)=0.025$. In other words, the maximum sugar ratio that can be measured is uttermost 2.5\%. Calibration of the MA871 digital Brix refractometer was also performed according to these ratios.

4.2. Results of Mixing Experiments. The homogeneity ratio obtained at the end of the mixing experiment done by using the Delta robot-based chaotic mixer and the Sprott_A system with the highest ODR is shown in Table 7.

The homogeneity ratio obtained at the end of the mixing experiment carried out by using the Delta robot-based chaotic mixer and the Lu-Chen system with the lowest ODR is shown in Table 8.

The homogeneity ratio obtained at the end of the mixing experiment done with the circular motion system and the Delta robot based chaotic mixer is as shown in Table 9. 
TABle 3: Comparison of chaotic systems and circular motion ODR.

\begin{tabular}{|c|c|c|c|c|c|c|c|c|c|c|c|}
\hline Systems & $\begin{array}{l}\text { Time } \\
\text { (Min.) }\end{array}$ & $\begin{array}{c}\text { Zone- } \\
1 \%\end{array}$ & $\begin{array}{c}\text { Zone- } \\
2 \% \\
\end{array}$ & $\begin{array}{c}\text { Zone- } \\
3 \%\end{array}$ & $\begin{array}{c}\text { Zone- } \\
4 \% \\
\end{array}$ & $\begin{array}{c}\text { Zone- } \\
5 \% \\
\end{array}$ & $\begin{array}{c}\text { Zone- } \\
6 \% \\
\end{array}$ & $\begin{array}{c}\text { Zone- } \\
7 \%\end{array}$ & $\begin{array}{c}\text { Zone- } \\
8 \%\end{array}$ & $\begin{array}{c}\text { Total } \\
\%\end{array}$ & $\begin{array}{c}\text { Orbital } \\
\text { distribution rates }\end{array}$ \\
\hline Orbital & 5 & 0 & 0 & 0 & 0 & 24,90 & 24,90 & 25,10 & 25,10 & 100,0 & 42,9 \\
\hline Sprott A & 5 & 13,1 & 14,1 & 13,9 & 14,4 & 10,9 & 10,7 & 11,4 & 11,5 & 100,0 & 93,7 \\
\hline Pehlivan-Wei & 5 & 4,4 & 12,6 & 13,8 & 33,4 & 0,7 & 10,7 & 8,4 & 16,0 & 100,0 & 70,5 \\
\hline Aizawa & 5 & 18,7 & 8,6 & 10,8 & 3,9 & 27,8 & 11,6 & 11,2 & 7,4 & 100,0 & 75,4 \\
\hline Guckenheimer-Holmes & 5 & 3,9 & 7,3 & 6,3 & 25,1 & 4,2 & 7,6 & 8,5 & 37,1 & 100,0 & 57,5 \\
\hline Lu-Chen & 5 & 0 & 0 & 0 & 0 & 46,3 & 16,1 & 9,8 & 27,5 & 100,0 & 40,1 \\
\hline Hadley & 5 & 0,0 & 23,0 & 3,6 & 19,8 & 0,8 & 29,8 & 0,4 & 22,6 & 100,0 & 48,3 \\
\hline Halvorsen & 5 & 0,4 & 20,6 & 21,2 & 11,0 & 21,1 & 11,6 & 12,5 & 1,6 & 100,0 & 71,0 \\
\hline
\end{tabular}

TABLE 4: ODR belonging to the Sprott-A chaotic system.

\begin{tabular}{lccccccccc}
\hline Zone-1\% & Zone-2\% & Zone-3\% & Zone-4\% & Zone-5\% & Zone-6\% & Zone-7\% & Zone-8\% & Total \% & Orbital distribution rates \\
\hline 13,1 & 14,1 & 13,9 & 14,4 & 10,9 & 10,7 & 11,4 & 11,5 & 100,0 & 93,7 \\
\hline
\end{tabular}

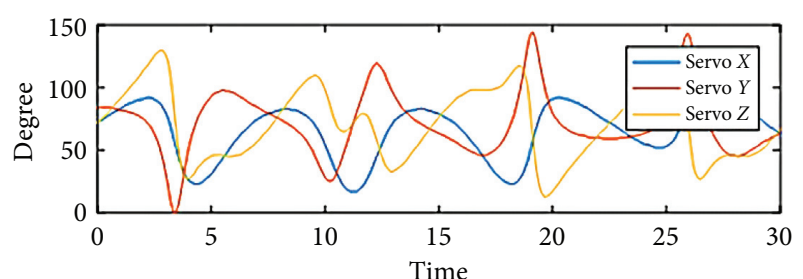

Figure 11: $x-y-z$ time series of the Sprott A system.

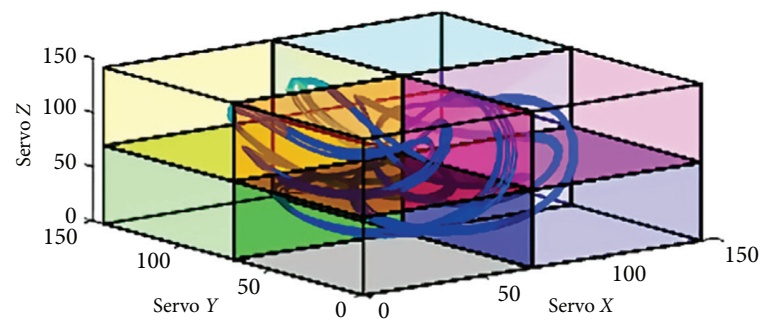

Figure 12: 3D phase portrait of the Sprott A system.

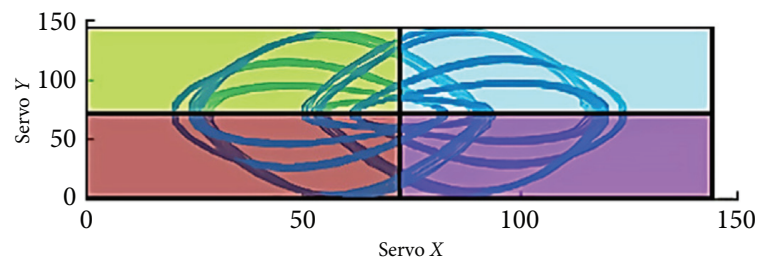

Figure 13: $x-y$ phase portrait of the Sprott A system.

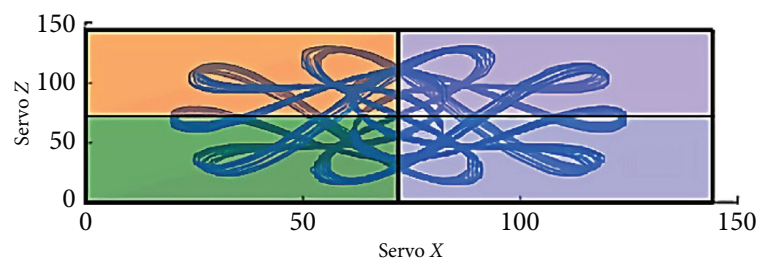

Figure 14: $x-z$ phase portrait of the Sprott A system.

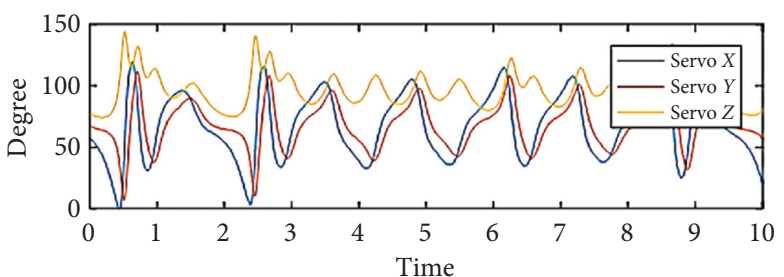

Figure 15: $x-y-z$ time series of the Lu-Chen system.

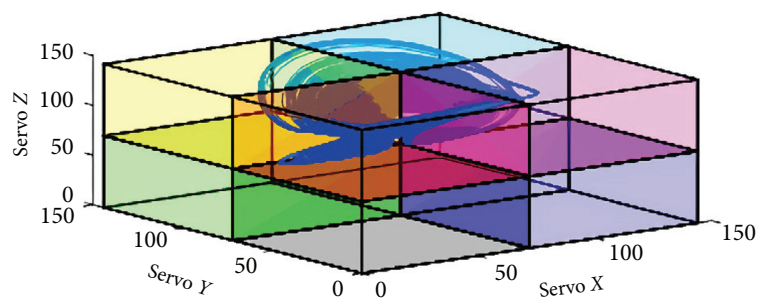

FIgURE 16: 3D phase portrait of the Lu-Chen system.

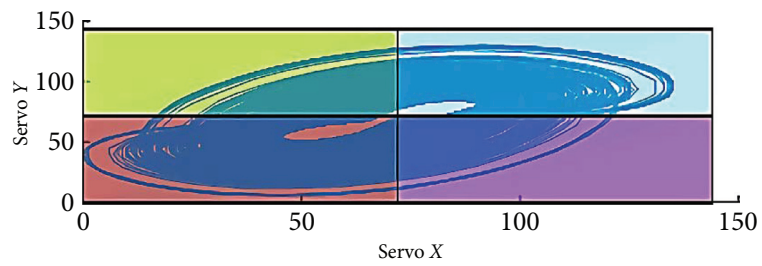

FIGURE 17: $x-y$ phase portrait of the Lu-Chen system.

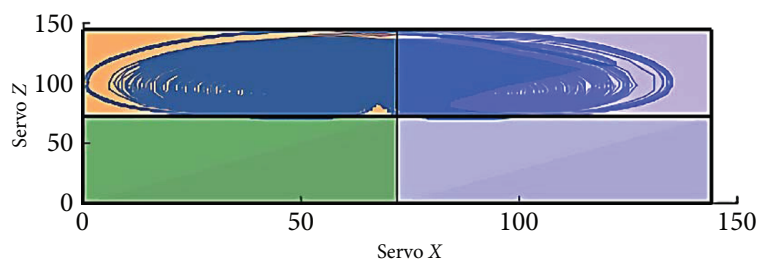

FIgURE 18: $x-z$ phase portrait of the Lu-Chen system. 
TABLE 5: ODR belonging to the Lu-Chen chaotic system.

\begin{tabular}{cccccccccc}
\hline Zone-1\% & Zone-2\% & Zone-3\% & Zone-4\% & Zone-5\% & Zone-6\% & Zone-7\% & Zone-8\% & Total \% & Orbital distribution rates \\
\hline 0 & 0 & 0 & 0 & 46,3 & 16,1 & 9,8 & 27,5 & 100,0 & 40,1
\end{tabular}

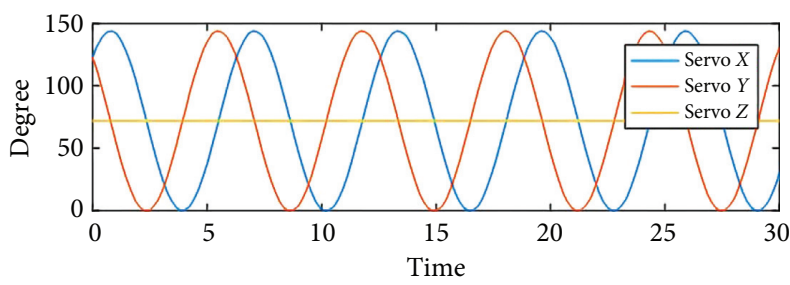

Figure 19: $x-y-z$ time series of the circular motion.

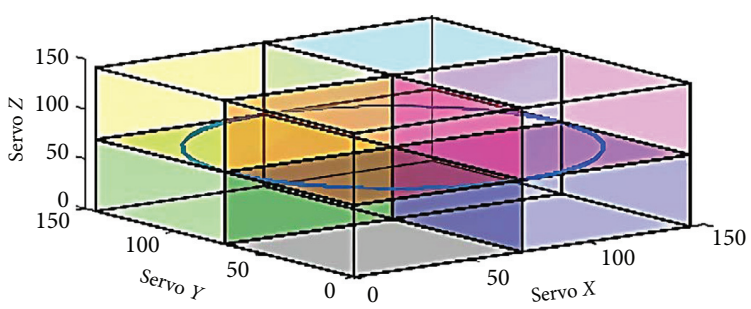

FiguRe 20: 3D phase portrait of the circular motion system.

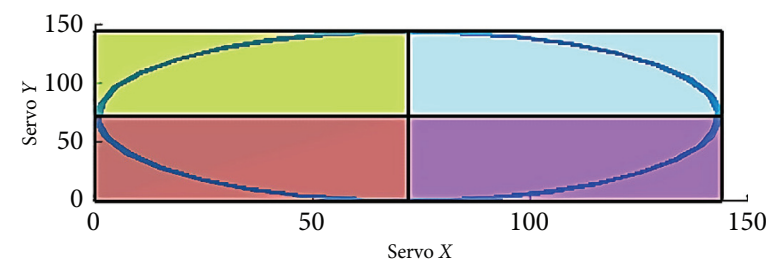

FIGURE 21: $x-y$ phase portrait of the circular motion system.

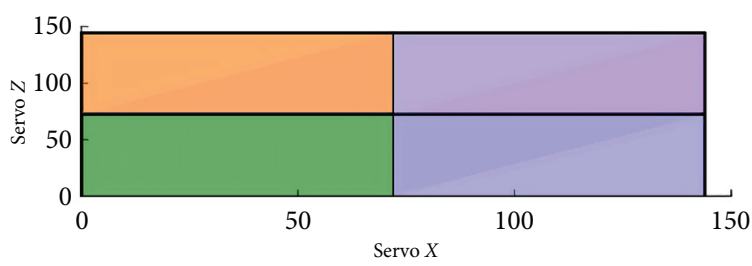

Figure 22: $x-z$ phase portrait of the circular motion system.

TABLE 6: Circular motion ODR.

\begin{tabular}{cccccccccc}
\hline Zone-1\% & Zone-2\% & Zone-3\% & Zone-4\% & Zone-5\% & Zone-6\% & Zone-7\% & Zone-8\% & Total \% & Orbital distribution rates \\
\hline 0 & 0 & 0 & 0 & 24,90 & 24,90 & 25,10 & 25,10 & 100,0 & 42,9 \\
\hline
\end{tabular}

The homogeneity ratio obtained at the end of the mixing experiment performed with the constant motion of the propeller mixer is shown in Table 10.

When the homogeneity ratios obtained at the end of 4 different mixing tests are checked (Tables 7-10), it was seen that the Sprott_A system had the highest homogeneity measurement ratio with $72 \%$. The homogeneity ratio was measured $60 \%$ in the $\mathrm{Lu}-\mathrm{Chen}$ system, $64 \%$ in the circular motion system, and $52 \%$ in the constant motion of the propeller. 


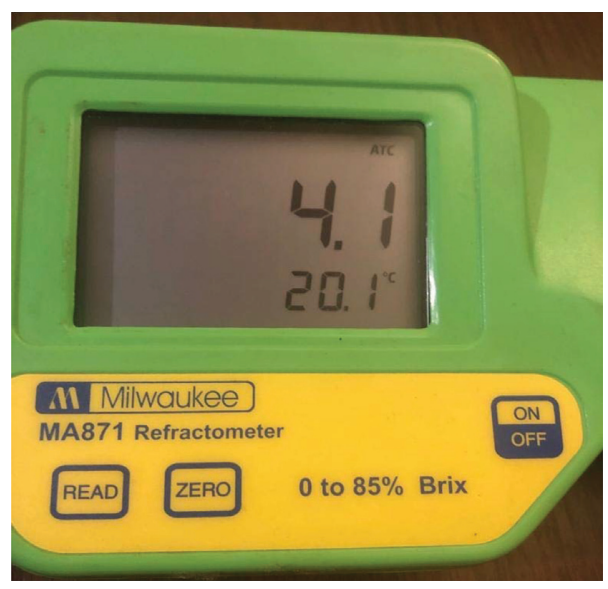

Figure 23: MA871 digital Brix refractometer.

TABLE 7: The homogeneity rate obtained in the mixing experiment done with the Sprott_A chaotic system.

\begin{tabular}{cccccccccccc}
\hline Zone-1\% & Zone-2\% & Zone-3\% & Zone-4\% & Zone-5\% & Zone-6\% & Zone-7\% & Zone-8\% & $\begin{array}{c}\text { Total } \\
\%\end{array}$ & $\begin{array}{c}\text { Orbital } \\
\text { distribution } \\
\text { rates }\end{array}$ & $\begin{array}{c}\text { Measured } \\
\text { sugar rate }\end{array}$ & $\begin{array}{c}\text { Homogeneity } \\
\text { rate }\end{array}$ \\
\hline 13,1 & 14,1 & 13,9 & 14,4 & 10,9 & 10,7 & 11,4 & 11,5 & 100,0 & 93,7 & 1,8 & 72,0 \\
\hline
\end{tabular}

TABle 8: The homogeneity rate obtained in the mixing experiment done with the Lu-Chen chaotic system.

\begin{tabular}{cccccccccccc}
\hline Zone-1\% & Zone-2\% & Zone-3\% & Zone-4\% & Zone-5\% & Zone-6\% & Zone-7\% & Zone-8\% & $\begin{array}{c}\text { Total } \\
\%\end{array}$ & $\begin{array}{c}\text { Orbital } \\
\text { distribution } \\
\text { rates }\end{array}$ & $\begin{array}{c}\text { Measured } \\
\text { sugar rate }\end{array}$ & $\begin{array}{c}\text { Homogeneity } \\
\text { rate }\end{array}$ \\
\hline 0 & 0 & 0 & 0 & 46,3 & 16,1 & 9,8 & 27,5 & 100,0 & 40,1 & 1,5 & 60,0 \\
\hline
\end{tabular}

TABLE 9: The homogeneity rate obtained in the mixing experiment done with the circular motion system.

\begin{tabular}{cccccccccccc}
\hline Zone-1\% & Zone-2\% & Zone-3\% & Zone-4\% & Zone-5\% & Zone-6\% & Zone-7\% & Zone-8\% & $\begin{array}{c}\text { Total } \\
\%\end{array}$ & $\begin{array}{c}\text { Orbital } \\
\text { distribution } \\
\text { rates }\end{array}$ & $\begin{array}{c}\text { Measured } \\
\text { sugar rate }\end{array}$ & $\begin{array}{c}\text { Homogeneity } \\
\text { rate }\end{array}$ \\
\hline 0 & 0 & 0 & 0 & 24,90 & 24,90 & 25,10 & 25,10 & 100,0 & 42,9 & 1,6 & 64,0 \\
\hline
\end{tabular}

TABle 10: The homogeneity rate obtained in the mixing experiment performed with the constant motion of the propeller mixer.

\begin{tabular}{cccccccccccc}
\hline Zone-1\% & Zone-2\% & Zone-3\% & Zone-4\% & Zone-5\% & Zone-6\% & Zone-7\% & Zone-8\% & $\begin{array}{c}\text { Total } \\
\%\end{array}$ & $\begin{array}{c}\text { Orbital } \\
\text { distribution } \\
\text { rates }\end{array}$ & $\begin{array}{c}\text { Measured } \\
\text { sugar rate }\end{array}$ & $\begin{array}{c}\text { Homogeneity } \\
\text { rate }\end{array}$ \\
\hline 0 & 0 & 0 & 0 & 0 & 0 & 0 & 0 & 0 & 0 & 1,3 \\
\hline
\end{tabular}

\section{Conclusions}

This study had been carried out by designing a new delta robot-based chaotic mixer. With the algorithm developed in MATLAB, the amplitude values of time series produced from chaotic systems were converted to 3-dimensional position information for servo motors used in the mixer. The chaotic position information was transferred to the Arduino Uno R3 card allowing the newly designed chaotic mixer to be checked to pace chaotically on the $x, y$, and $z$ coordinates.

With the software developed in MATLAB, the performances of trajectories which belong to 7 different chaotic systems and the performances of circular motion trajectories were evaluated by comparing them over the numerical simulation ODRs. According to the results, the Sprott_A system was the chaotic system with the highest ODR of 93.7\%, while the Lu-Chen system was the chaotic system with the lowest ODR of $40.1 \%$. The circular motion ODR was found out as $42.9 \%$.

Mixing experiments were carried out for the Sprott_A system with the highest ODR, Lu-Chen system with the lowest ODR, and mixing propeller's constant and circular motion movement. 5-minute periods were selected for all 4 different experimental studies. Due to the physical structure of the chaotic mixer, 20 liters of solutions, consisting of 19.5 liters of water and 0.5 liters of sugar were prepared for each experiment. The MA871 digital Brix refractometer measurement device was used to measure the homogeneity 
rate of the solution in the mixing tests. As a result of the experiments, the Sprott_A system was the system with the highest homogeneity measurement ratio with $72 \%$. The $\mathrm{Lu}-\mathrm{Chen}$ system was $60 \%$, the circular motion system was $64 \%$, and the constant motion of the propeller was $52 \%$. The system with the highest homogeneity measurement ratio was the Sprott_A system as in the ODR calculations. It has been observed that even the Lu-Chen chaotic system with the lowest ODR yields better results than the constant moving mixing process.

When evaluated in terms of mixing time, it was found that the Sprott A system achieved the same uniformity ratio in $20 \%$ less time than the circular motion mixing process and $40 \%$ less than the fixed motion process.

It is thought that this prototype presented in the article can serve the purpose of developing new chaotic mixer systems and algorithms that can produce homogeneous mixtures of the same quality with less working time and less power consumption instead of the fixed and circular moving mixers widely used today.

In the next study, using artificial intelligence techniques, it is aimed that the chaotic mixer automatically decides which chaotic system it will work with according to the state of the mixture.

The results obtained in this study will be helpful with other studies in the fields such as materials science and nanotechnology engineering [33, 67], 3D printing technology [68], biological fluid dynamics [69], medical/biomedical sciences, and food and chemical industries [70].

\section{Data Availability}

No data were used to support this study.

\section{Conflicts of Interest}

The authors declare that they have no conflicts of interest.

\section{References}

[1] E. Kurt, A New Chaotic Mixer Design and Application, Sakarya University, Institute of Natural Sciences, Sakarya, Turkey, 2017.

[2] J. T. Banhero and W. L. Bodger, Introduction to Chemical Engineering, pp. 731-737, ITU Publications, Geneva, Switzerland, 1979.

[3] W. Vauck and H. Müler, Basic Operations of Chemical Engineering Introduction, pp. 307-328, Theodor Steinkopff, Magdeburg, Germany, 1966.

[4] J. Henzler and H. Eignung, Von Kontinuierlich Durchströmten Mischern Zum Homogenisieren, pp. 1-8, WILEY-VCH publications, Hoboken, NJ, USA, 1972.

[5] N. Ilten, Investigation of Current Events in Mixers and Mixing Vessel, Uludağ University, Institute of Natural Sciences, Department of Mechanical Engineering, Bursa, Turkey, 1986.

[6] B. A. Can, Mixing of Different Fluids in the Mixing Vessel and Analysis of the Temperature Distribution, Erciyes University, Institute of Natural Sciences, Department of Mechanical Engineering, Kayseri, Turkey, 2010.

[7] T. Dodok, N. Čuboňová, M. Císar, I. Kuric, and I. Zajačko, "Utilization of strategies to generate and optimize machining sequences in CAD/CAM," Procedia Engineering, vol. 192, pp. 113-118, 2017.

[8] P. Bozek and Y. Turygin, "Measurement of the operating parameters and numerical analysis of the mechanical subsystem," Measurement Science Review, vol. 14, no. 4, 2014.

[9] P. Bozek, A. A. Akkad, P. Blistan, and I. Ibrahim, "Navigation control and stability investigation of a mobile robot based on a hexacopter equipped with an integrated manipulator," International Journal of Advanced Robotic Systems, vol. 14, no. 6, pp. 1-13, 2017.

[10] A. Kilin, P. Bozek, Y. Karavaev, A. Klekovkin, and V. Shestakov, "Experimental investigations of a highly maneuverable mobile omniwheel robot," International Journal of Advanced Robotic Systems, vol. 14, no. 6, pp. 1-9, 2017.

[11] J. Angeles, Fundamentals of Robotic Mechanical Systems: Theory, Methods and Algorithms, Springer, Berlin, Germany, 1997.

[12] P. J. Zsombo-Murray, Descriptive Geometric Kinematic Analysis of Clavel's "Delta" Robot, McGill University, Department of Mechanical Engineering, Centre for Intelligent Machines, Montreal, Canada, 2004.

[13] I. H. Sanliturk, Investigation of Delta Robot Design and Performance Characteristics, Firat University, Institute of Natural Sciences, Department of Mechanical Engineering, Elazı ğ, Turkey, 2012.

[14] Y. Qiaoling, J. Shiming, W. Zhongfei, W. Guan, W. Yuehua, and Z. Li, "Optimal design of the linear delta robot for prescribed cuboid dexterous workspace based on performance chart," in Proceedings of the 8th WSEAS International Conference on Robotics, Control And Manufacturing Technology (Rocom'08), pp. 35-41, Hangzhou, China, April 2008.

[15] L. Xin-Jun, W. Jinsong, O. Kun-Ku, and K. Jongwon, "A new approach to the design of a delta robot with the desired workspace," Journal of Intelligent and Robotic Systems, vol. 39, pp. 209-225, 2004.

[16] U. Cavusoglu, Y. Uyaroğlu, and İ. Pehlivan, "Design of a continuous-time autonomous chaotic circuit and application of signal masking," Journal of the Faculty of Engineering and Architecture of Gazi University, vol. 29, no. 1, pp. 79-87, 2014.

[17] I. Koyuncu, A. Ozcerit, and I. Pehlivan, "An analog Ccircuit design and FPGA-based implementation of the burke-shaw chaotic system," Optoelectronics and Advanced Materials-Rapid Communications, vol. 7, no. 9-10, pp. 635-638, 2013.

[18] L. Chunbiao, I. Pehlivan, and J. C. Sprott, "Amplitude-phase control of a novel chaotic attractor," Turkish Journal of Electrical Engineering \& Computer Sciences, vol. 24, no. 1, pp. 1-11, 2016.

[19] I. Pehlivan, Y. Uyaroglu, and M. Yogun, "Chaotic oscillator design and realizations of the rucklidge attractor and its synchronization and masking simulations," Scientific Research and Essays, vol. 5, no. 16, pp. 2210-2219, 2010.

[20] M. Alcin, I. Pehlivan, and I. Koyuncu, "Hardware design and implementation of a novel ANN-based chaotic generator in FPGA," OPTIK-international Journal for Light and Electron Optics, vol. 127, no. 13, pp. 5500-5505, July 2016.

[21] M. Alcin, I. Koyuncu, M. Tuna, M. Varan, and I. Pehlivan, “A novel high speed artificial neural network-based chaotic true random number generator on field programmable gate array," International Journal of Circuit Theory and Applications, vol. 47, no. 3, 2018.

[22] U. Cavusoglu, S. Kacar, A. Zengin, and I. Pehlivan, "A novel hybrid encryption algorithm based on chaos and S-AES 
algorithm," Nonlinear Dynamics, vol. 92, no. 4, pp. 1745-1759, 2018.

[23] U. Cavusoglu, A. Akgul, A. Zengin, and I. Pehlivan, "The design and implementation of hybrid RSA algorithm using a novel chaos based RNG," Chaos, Solitons \& Fractals, vol. 104, pp. 655-667, 2017.

[24] U. Cavusoglu, A. Zengin, I. Pehlivan, and S. Kacar, "A novel approach for strong S-Box generation algorithm design based on chaotic scaled zhongtang system," Nonlinear Dynamics, vol. 87, no. 2, pp. 1081-1094, 2017.

[25] A. Ozdemir, I. Pehlivan, A. Akgül, and E. Güleryüz, "A strange novel chaotic system with fully golden proportion equilibria and its mobile microcomputer-based RNG application," Chinese Journal of Physics, vol. 56, no. 6, pp. 2852-2864, 2018.

[26] A. Akgul, C. Arslan, and B. Aricilioglu, "Design of an interface for random number generators based on integer and fractional order chaotic systems," Chaos Theory and Applications, vol. 1, no. 1, pp. 1-18, 2019.

[27] I. Pehlivan, K. Ersin, L. Qiang, A. Basaran, and M. Kutlu, “A multi scroll chaotic attractor and its electronic circuit implementation," Chaos Theory and Applications, vol. 1, no. 1, pp. 29-37, 2019.

[28] K. T. Chau, Y. Shuang, G. Yuan, and J. H. Chen, "Application of chaotic-motion motors to industrial mixing processes," IAS2004, pp. 1874-1880, Wiley, Hoboken, NJ, USA, 2004.

[29] S. Ye and K. T. Chau, "Destabilization control of a chaotic motor for industrial mixers," IAS2005, pp. 1724-1730, Wiley, Hoboken, NJ, USA, 2005.

[30] S. Ye and K. T. Chau, "Chaoization of DC motors for industrial mixing," IEEE Transactions on Industrial Electronics, vol. 54, no. 4, 2007.

[31] M. A. Murtadha, M. Abdurrahman, and A. I. Korman, Chaotic Control of Liquid Mixer, The University of Sharjah, Department Of Electrical \& Computer Engineering. Senior Design Project II, Sharjah, UAE, 2008.

[32] Z. Zhang and G. Chen, "Liquid mixing enhancement by chaotic perturbations in stirred tanks," Chaos, Solitons \& Fractals, vol. 36, no. 1, pp. 144-149, 2008.

[33] A. E. Kavur, S. Demiroğlu, M. Ö. Seydibeyoğlu, Ö. Baser, C. Güzelis, and S. Şahin, "Design and implementation of chaotic system based robust delta robot for blending graphene nanoplatelets," in Proceedings of the 2016 21st International Conference on Methods and Models in Automation and Robotics (MMAR), IEEE, Miedzyzdroje, Poland, August 2016.

[34] I. Pehlivan, New Chaotic Systems: Electronic Circuit Realizations, Synchronization and Secure Communication Applications, Sakarya University, Institute of Natural Sciences, Department of Electrical and Electronics Engineering, Sakarya, Turkey, 2007.

[35] P. S. Addison, Fractals and Chaos, an Illustrated Course, pp. 5-7, IOP Publishing Limited, Bristol, UK, 1997.

[36] R. C. Hilborn, "An introduction for scientists and engineers," Chaos and Nonlinear Dynamics, Oxford University Press, Oxford, UK, 1994.

[37] I. Koyuncu, Design and Implementation of FPGA-Based New Chaotic Oscillators and Real Random Number Generators for Cryptological Applications, Sakarya University, Institute of Natural Sciences, Sakarya, Turkey, 2014.

[38] A. Akgul, Random Number Generator Design with New Chaotic Systems and High Security Encryption of Multimedia Data, Sakarya University, Institute of Natural Sciences, Sakarya, Turkey, 2015.
[39] S. Ozoguz and A. Zeki, "Integrated implementation of continuous time chaotic systems and their use in random number generation," Tubitak Project Final Report, (106E093), 2008.

[40] K. Ozdemir, Random Number Generator Design with Continuous Time Chaos, Istanbul Technical University, Istanbul, Turkey, 2008.

[41] S. Coskun, Design and Implementation of Chaos Sourced and Adc Based Novel True Random Number Generators, Sakarya University, Institute of Natural Sciences, Department of Electrical and Electronics Education, Sakarya, Turkey, 2017.

[42] N. K. Pareek, V. Patidar, and K. K. Sud, "Image encryption using chaotic logistic map," Image and Vision Computing, vol. 24, no. 9, pp. 926-934, 2006.

[43] F. E. Udwadia and R. S. Guttalu, "Chaotic Dynamics of a piecewise cubic map," Physical Review A, vol. 40, no. 7, pp. 4032-4044, 1989.

[44] B. Saha, S. Tej Malasani, and J. B. Seventline, "Application of modified chaotic sine map in secure communication," International Journal of Computer Applications, vol. 113, no. 13, pp. 9-13, 2015.

[45] A. Vladi, A. Luca, O. Hodea, and R. Tataaru, Generating Chatic Secure Sequence Using Tent Map and Running-Key Approach, pp. 295-302, The Publishing House of The Romanian Academy, Bucharest, Romania, 2013.

[46] B. F. Vajargah and R. Asghari, "A pseudo random number generator based on chaotic henon map (CHCG)," International Journal of Mechatronics, Electrical and Computer Technology (IJMEC), vol. 5, no. 15, pp. 2120-2129, 2015.

[47] M. A. Aziz-Alaoui, C. Robert, and C. Grebogi, "Dynamics of a Hénon-Lozi-type map," Chaos, Solitons \& Fractals, vol. 12, no. 12, pp. 2323-2341, 2001.

[48] R. Senkerik, I. Zelinka, M. Pluhacek, and Z. K. Oplatkova, "Evolutionary control of chaotic burgers map by means of chaos enhanced differential evolution," International Journal of Mathematics and Computers in Simulation, vol. 8, pp. 39-45, 2014.

[49] S. M. Sohel Rana, "Chaotic dynamics in a discrete-time predator-prey food chain," Computational Ecology and Software, vol. 5, no. 1, pp. 28-47, 2015.

[50] M. Mishra, A. Ranjan Routray, and S. Kumar, "High security image steganography with modified Arnold's Cat map," International Journal of Computer Applications, vol. 37, no. 9, pp. 16-20, 2012.

[51] J. C. Butcher, Numerical Methods for Ordinary Differential Equations, John Wiley \& Sons, Hoboken, NJ, USA, 2008.

[52] W. Zhang, Z. Gui, and K. Wang, "Impulsive control for synchronization of lorenz chaotic system," Journal of Software Engineering and Applications, vol. 5, no. 12, pp. 23-25, 2013.

[53] R.-E. Precup, M.-L. Tomescu, and C.-A. Dragos, "Stabilization of Rössler chaotic dynamical system using fuzzy logic control algorithm," International Journal of General Systems, vol. 43, no. 5, pp. 413-433, 2014.

[54] L.-J. Sheu, H.-K. Chen, J.-H. Chen, and L.-M. Tam, "Chaotic dynamics of the fractionally damped Duffing equation," Chaos, Solitons \& Fractals, vol. 32, no. 4, pp. 1459-1468, 2007.

[55] M. Kuetche, E. S. Fotsin, H. B. Kengne, and P. Woafo, "“Parameters estimation based adaptive GPS of chaotic Chua's circuit with application to chaos communication by parametric modulation," Chaos, Solitons \& Fract, pp. 27-37, Elsevier, Amsterdam, Netherlands, 2014.

[56] V. Sundarapandian, "Global chaos synchronization of the forced van der pol chaotic oscillators via adaptive control method," International Journal of Pharm Tech Research, vol. 8, no. 6, pp. 156-166, 2015. 
[57] G. Chen and T. Ueta, Chaos in Circuits and Systems, World Scientific Pub. Co., Singapore, 2002.

[58] V. Vembarasan and P. Balasubramaniam, "Chaotic synchronization of Rikitake system based on T-S fuzzy control techniques," Nonlinear Dynamics, vol. 74, no. 1-2, pp. 31-44, 2013.

[59] A. M. Rucklidge, "Chaos in models of double convection," Journal of Fluid Mechanics, vol. 237, pp. 209-229, 1992.

[60] V. Sundarapandian, "Global chaos synchronization of rucklidge chaotic systems for double convection via sliding mode control," International Journal of ChemTech Research, vol. 8, no. 8, pp. 61-72, 2015.

[61] V. Sundarapandian, "Output regulation of the arneodo chaotic system," International Journal on Computer Science and Engineering (IJCSE), vol. 2, no. 5, pp. 1601-1608, 2010.

[62] Z.-L. Wang and X.-R. Shi, "Chaotic bursting lag synchronization of hindmarsh-rose system via a single controller," Applied Mathematics and Computation, vol. 215, no. 3, pp. 1091-1097, 2009.

[63] W. Zhongtang, M. Wang, J. Jianxiu, and F. Jiuchap, "A Novel strange attractor and its dynamic analysis," Journal of Multimedia, vol. 9, no. 3, pp. 408-415, 2014.

[64] R. Neugebauer, V. Wittstock, and W. Drossel, Werkzeugmaschinen Mechatronic, Chemnitz University of Technology, Chemnitz, Germany, 2010.

[65] E. Ozkilic, Parametrical Simulation of 3 Freedom Delta Robot Mechanisms with Matlab-Simmechanics, Yildiz Technical University, Institute of Natural Sciences, Department of Mechanical Engineering, Istanbul, Turkey, 2012.

[66] http://www.milwaukeeinst.com/site/products/products/ digital-refractometers/165-products-g-digitalrefractometers-g-ma871.

[67] S. Şahin, A. E. Kavur, S. D. Mustafov et al., "Spatiotemporal chaotification of delta robot mixer for homogeneous graphene nanocomposite dispersing," Robotics and Autonomous Systems, vol. 134, 2020.

[68] D. Therriault, S. R. White, and J. A. Lewis, "Chaotic mixing in three-dimensional microvascular networks fabricated by direct-write assembly," Nature Materials, vol. 2, no. 4, pp. 265-271, 2003.

[69] M. A. Jalali, A. Khoshnood, and M.-R. Alam, "Microswimmer-induced chaotic mixing," Journal of Fluid Mechanics, vol. 779, pp. 669-683, 2015.

[70] S. K. Lao, H. K. Chen, L. M. Tam, and L. J. Sheu, "Microfluidic mixing using chaotic signals from the Chen-Lee system," in Proceedings of the International Conference on Advanced Robotics and Intelligent Systems (ARIS), Taipei, Taiwan, May 2015. 